\title{
A trait-like propensity to experience internalizing symptoms is associated with problem alcohol involvement across adulthood, but not adolescence
}

\author{
Kevin M. King ${ }^{1}$, Madison C. Feil ${ }^{1}$, Max A. Halvorson ${ }^{1}$, Rick Kosterman ${ }^{2}$, Jennifer A. Bailey ${ }^{2}$, \\ J. David Hawkins ${ }^{2}$ \\ 1Department of Psychology, University of Washington \\ ${ }^{2}$ Social Development Research Group, School of Social Work, University of Washington
}

\begin{abstract}
There are stable between-person differences in an internalizing "trait," or the propensity to experience symptoms of internalizing disorders, such as social anxiety, generalized anxiety disorder, and depression. Trait internalizing may serve as a marker of heightened risk for problem alcohol outcomes (such as heavier drinking, binge drinking or alcohol dependence). However, prior research on the association between internalizing symptoms and alcohol outcomes has been largely mixed in adolescence, with more consistent support for an association during adulthood. It may be that trait internalizing is only associated with problem alcohol outcomes in adulthood, after individuals have gained experience with alcohol. Some evidence suggested that these effects may be stronger for females than males. We used data from a community sample $(n=790)$ interviewed during adolescence (ages $14-16$ ) and again at ages 21, 24, 27, 30, 33, and 39. Using generalized estimating equations, we tested the association between trait internalizing and alcohol outcomes during both adolescence and adulthood, and tested whether adult trait internalizing mediated the association between adolescent trait internalizing and adult alcohol outcomes. Trait internalizing in adulthood (but not adolescence) was associated with more frequent alcohol use, binge drinking and symptoms of alcohol use disorders, and mediated the effects of adolescent trait internalizing on alcohol outcomes. We observed no moderation by gender or change in these associations over time. Understanding the developmental pathways of trait internalizing may provide further insights into preventing the emergence of problem alcohol use behavior during adulthood.
\end{abstract}

\section{Keywords}

internalizing; alcohol use disorders; development; generalized estimating equations; comorbidity

Correspondence concerning this article should be addressed to Kevin M. King, Department of Psychology, University of Washington. kingkm@uw.edu. 


\section{Introduction}

Theories of the role of internalizing symptoms in the development of alcohol outcomes (such as use, binge drinking and symptoms of alcohol use disorders; AUDs) hypothesize that the experience of internalizing symptoms influences alcohol outcomes through negative reinforcement mechanisms (Cloninger, 1987; Hussong et al., 2011; Koob \& Le Moal, 2008; Sher, 1991; Wills \& Filer, 1996). However, tests of this hypothesis among adolescents have received mixed empirical support (Chassin et al., 2003; Hussong et al., 2017; Hussong et al., 2011). This may be in part because prior studies failed to disaggregate the effects of individual differences in the tendency to experience internalizing symptoms from the effects of episodic increases in internalizing symptoms on alcohol use. Studies are needed that separate between-person from within-person variance in internalizing symptoms (Curran \& Bauer, 2011; King et al., 2018).

\section{A Propensity Towards Internalizing Symptoms as a Risk for AUDs}

Considerably less attention has been paid to testing whether individual differences in a propensity to experience internalizing symptoms are associated with the development of problem alcohol outcomes. The goal of this article is to leverage longitudinal betweenperson data to test an explicitly between-person hypothesis: that some individuals are more likely to develop problem alcohol outcomes because they are prone to experience symptoms of internalizing disorders. Theories of "trait internalizing" suggest that some individuals are more likely to report symptoms of and meet criteria for multiple internalizing disorders such as major depression, generalized anxiety disorder, social phobia and others across time (Eaton et al., 2011; Krueger \& Markon, 2006). Theories posit that this tendency exhibits a high degree of stability across adulthood (Eaton et al., 2011). Trait internalizing is thought to emerge from negative emotionality, a biologically based predisposition towards frequent and intense negative emotions in childhood (Barlow et al., 2014; Griffith et al., 2010), which has long been implicated in the development of AUDs.

Two prior studies found that trait internalizing was associated with risk for AUDs in adulthood. First, a study using a nationally representative sample of adults found that trait internalizing across comorbid conditions was moderately associated with both past year $(r$ $=.38)$ and lifetime $(r=.49)$ alcohol dependence (Kushner et al., 2012). Another study showed that trait internalizing was associated with the tendency to meet criteria for AUDs during young adulthood, between ages 18 and 29 ( $r=.43$; Jackson \& Sher, 2003). However, those studies did not include measures of internalizing symptoms or alcohol use behaviors during adolescence. In the current manuscript, we aimed to integrate models of comorbidity among internalizing problems with models of the developmental psychopathology of alcohol outcomes in order to better understand who is at risk, and when that risk is most likely to be observed.

\section{The Importance of Developmental Timing}

The association between trait internalizing and alcohol use behaviors (including AUDs) may depend on the developmental period where this association is studied. Much of the prior research on internalizing symptoms and alcohol outcomes focused on relatively early 
developmental stages such as adolescence and young adulthood, when alcohol use is often just beginning. Results of this research have been mixed or null (Farmer et al., 2016; Fleming et al., 2008; Hussong et al., 1998; Kaplow et al., 2002; King \& Chassin, 2008; S. M. King et al., 2004; Sihvola et al., 2008). Although little of this research focused on common variance among internalizing symptoms, a recent review showed that most studies reported null effects (Hussong et al., 2017; Hussong et al., 2011). In one national study, only $61.5 \%$ of high school seniors reported any lifetime alcohol use (Johnston et al., 2016), suggesting that many adolescents are either just beginning to drink alcohol or have not yet initiated drinking. When alcohol use does escalate in adolescence, it is largely driven by involvement in deviant peer groups and externalizing symptoms, which may overshadow the importance of internalizing symptoms in this age range (Colder et al., 2017). Further, because some internalizing symptoms are characterized by heightened behavioral inhibition, it is possible that trait internalizing may actually be protective against alcohol use in adolescence by delaying experiences with alcohol (Colder et al., 2013). For example, two studies recently demonstrated that trait internalizing was largely protective against alcohol use during adolescence ( ages 11 to 19 ) when controlling for externalizing symptoms and that the highest likelihood and level of alcohol use was observed for those with low internalizing symptoms and high externalizing symptoms (Colder et al., 2018, 2017).. Importantly, one of those studies (Colder et al., 2017) showed that the protective effects of trait internalizing on alcohol outcomes diminished with age, largely disappearing by age 19 .

In summary, trait internalizing appears to be associated with problem alcohol outcomes in adulthood, but not adolescence. Yet, trait internalizing is thought to be relatively stable across these developmental periods. Given the current literature, it is still unclear how early manifestations of trait internalizing may increase risk for later alcohol problems in adulthood. It may be that trait internalizing in adolescents, while not directly leading to increased alcohol use during that time period, may lay the groundwork for the emergence of problem alcohol outcomes (such as heavier alcohol use, more binge drinking and more AUD symptoms; Hussong et al., 2011) in adulthood because they reflect a vulnerability towards internalizing symptoms that is stable across the lifespan. With assessments in adolescence, as well as adulthood (from ages 21 through 39 ), the current study is an attempt to test this hypothesis.

\section{Gender Differences in AUD Etiology}

There is substantial evidence for gender differences in the etiology of alcohol outcomes, mean differences in risk exposure, and differential associations between risk factors and alcohol use versus alcohol problems. Overall, women drink less alcohol and experience fewer problems from their alcohol use than men (Kessler et al., 2005). Moreover, some important risk factors for excessive drinking (e.g., aggressiveness, impulsivity, sensation seeking) are less prevalent in women, and certain protective factors (e.g., perception of social sanctions for drinking, nurturance) are more prevalent in women (e.g., Blume, 1991; Chassin et al., 1985; Sher, 1991; for a review see Nolen-Hoeksema, 2004). Despite this generally more benign risk profile, women tend to experience some consequences of heavy alcohol use more acutely than men, especially in the domains of assault risk and harms to reproductive health (Blume, 1991; Klassen \& Wilsnack, 1986). There is evidence that there 
may be stronger links between the risk factors that women do experience and problematic alcohol outcomes (Foster et al., 2015). This may be especially true for risks stemming from internalizing symptoms given that women generally exhibit higher levels of internalizing symptoms and disorders than men (Kessler et al., 2005). Some research has shown that internalizing symptoms had stronger associations with problematic alcohol involvement for women vs. men. For example, in a nationally representative sample, comorbid internalizing and externalizing disorders were more strongly associated with alcohol use and alcohol use disorders for women than men (Dawson et al., 2010). Thus, there is reason to believe that there may be stronger associations between trait internalizing and risk for alcohol outcomes for women than for men. To address this potential difference, the current study integrates tests of moderation by biological sex throughout our analyses, because we did not have measures of gender or gender identity in the current study.

\section{Current Objectives}

We used data from age 14 to 39 to examine the associations of trait internalizing with alcohol use, binge drinking and AUD symptoms. We hypothesized that (1) trait internalizing would be unrelated to alcohol use during adolescence. We hypothesized that (2) trait internalizing would exhibit continuity across adolescence and adulthood. In adulthood, we hypothesized that (3) trait internalizing would be associated with more alcohol use, binge drinking and AUD symptoms, and that the association would strengthen over time. We hypothesized that (4) adult trait internalizing would mediate the association between adolescent trait internalizing and adult alcohol outcomes, and that (5) the predicted associations would be stronger among women than men.

\section{Methods}

\section{Sample}

The Seattle Social Development Project (SSDP) was constituted in 1985 from 18 Seattle public elementary schools that overrepresented students from high-crime neighborhoods ( $\mathrm{N}$ $=1,053)$. From this population, 808 students $(77 \%)$ consented to participate in the longitudinal study, beginning at age 10 . We used data from three waves in adolescence (ages 14-16; 2,424 total observations), and six waves during adulthood (ages 21, 24, 27, 30, 33, and 39; 14,544 total observations). Analyses for the current study included all participants who responded to at least one survey in adolescence $(n=780,96.5 \%$ of the total sample) or adulthood ( $n=767,95 \%$ of the total sample). There were no differences in baseline study variables between participants with and without missing data at any wave. The dataset was 49\% female and was 47\% European American, 26\% African American, 22\% Asian American, and 5\% Native American. Approximately 52\% of the sample experienced childhood poverty, as measured by eligibility for the federal free and reduced-price school lunch program at ages $10-13$. SSDP retention was $88 \%$ at minimum through the adult waves. A detailed summary of SSDP can be found in Hawkins et al. (1992). Data collection procedures were approved by the Human Subjects Review Committee of the University of Washington. SSDP included a multicomponent intervention in the elementary grades, consisting of teacher training, parenting classes, and social competence training for children. Prior analyses have found differences in the levels and prevalence of adult outcomes 
between SSDP intervention groups, including higher educational attainment and socioeconomic status, better mental health, less teen pregnancy, and fewer sexually transmitted infections among those receiving the full intervention compared to controls (Hawkins et al., 1999; Hawkins et al., 2001; Hawkins et al., 2005, 2008; Kosterman et al., 2019). With respect to the current analyses, we tested whether all main associations were moderated by treatment status, but found no evidence of moderation (all $p>.10$ ). Thus, we included individuals from both the intervention and control conditions and controlled for intervention status in all analyses.

\section{Measures}

Trait Internalizing-Because trait internalizing is characterized as individual differences in the propensity towards internalizing symptoms or disorders, we operationalized trait internalizing for each participant as their average internalizing symptoms across items, subscales and time. We were primarily interested in estimating between-person effects, such as how individual differences in "trait" internalizing were associated with levels and change in alcohol use during adulthood. Thus, we used grand-mean-centered person average scores to estimate trait internalizing during adolescence and adulthood (Enders \& Tofighi, 2007). Because prior research suggested that bias in the regression coefficient for an aggregated measure was a function of the number of measurements and its ICC (Lüdtke et al., 2008), we computed the reliability of our person-mean measures following the recommendations of Gottfredson (2018), (nobsICC)/(1+(nobs-1)ICC).

Adolescence.: Adolescent trait internalizing was measured using self-report of internalizing symptoms from the Youth Self-Report (YSR, Achenbach \& Edlebrock, 1993). At ages 14, 15 and 16, youth reported on 11 items from the Anxious/Depressed and Withdrawn/ Depressed subscales of the YSR, such as "I am nervous or tense," "I am too fearful or anxious," "I am withdrawn," and "I like to be alone." Response options ranged from $1=\mathrm{Not}$ at all true to $3=$ Often true. To compute a trait internalizing score, we computed a mean of all internalizing items at each age ( $a=.73-.77$; mean inter-item $r=0.21-0.25)$, and then computed a grand-mean centered average of internalizing symptoms across all ages ( $a$ $=.79$; mean inter-item $r=0.56$ ). This trait internalizing score was correlated $r=0.92$ with a latent factor of average internalizing symptoms across ages $14-16\left(\chi^{2}=.95, p=0.62\right.$, CFI $=1.00$, SRMR $=0.007$, RMSEA $=0.00$ ), and there was no evidence of change over time in trait internalizing during adolescence.

Adulthood.: Adult trait internalizing was measured using self-report of 6 symptoms of generalized anxiety disorder (GAD), 5 symptoms of social phobia and 9 symptoms of major depressive disorder (MDD), all at ages 21, 24, 27, 30, 33, and 39 with a modified version of the Diagnostic Interview Schedule (DIS) (Robins et al., 1981) which measured disorder criteria reported by participants in the past year specified by the DSM-IV (American Psychiatric Association, 1994). The DIS has been used frequently in studies of psychiatric disorders among adults drawn from the general population and has been demonstrated to be valid and reliable (Jaffee et al., 2002). To compute a trait internalizing score, we computed a count score reflecting the total number of GAD, social phobia and MDD symptoms endorsed in each year ( $a=.53-.66$ across waves; mean inter-item $r=0.34-0.41)$, and 
then computed a grand-mean centered average of these symptom counts across all years ( $a$ $=.80$, mean inter-item $r=0.40$ ). This trait internalizing score was correlated $r=.96$ with a latent factor of symptom counts across all years $\left(\chi^{2}=25.76, p=0.002, \mathrm{CFI}=0.98, \mathrm{SRM} R\right.$ $=0.032$, RMSEA $=0.049)$, and there was no evidence of change over time.

Next, we computed the reliability of trait internalizing measures in adolescence and adulthood. The ICC for trait internalizing during adolescence was .36; with 3 observations the estimated reliability was .79. The ICC for adult trait internalizing was .37; and the estimated reliability of adult trait internalizing with 6 observations was .77. In other words, $36 \%$ to $37 \%$ of the variance in internalizing symptoms over time was attributable to stable, between person variance, and the resulting trait internalizing measure produced a relatively reliable estimate of between person variability $(.77-.79)$. Together, these data supported the use of aggregated trait internalizing in adolescence and adulthood.

\section{Alcohol Use}

Adolescence.: Alcohol use was measured at ages $14-16$ with a single item asking participants to report the total number of times they consumed at least a full drink of alcohol in the past month. Participants, on average, reported alcohol use on approximately $52 \%$ of all measurements between ages 14 and 16 .

Adulthood.: Alcohol use was measured at ages 21, 24, 27, 30, 33, and 39 with a single item asking participants to report the total number of times they had consumed alcohol in the past month (i.e. "How many times did you drink alcohol in the past month?"). Participants, on average, reported alcohol use on approximately $63 \%$ of all measurements from ages $21-39$.

\section{Binge Drinking}

Adolescence.: Binge drinking was measured with a single item assessing how many times in the past month participants consumed 3 (for females) or 4 (for males) drinks in a row on a single occasion. Participants reported at least a single occasion of binge drinking on $14 \%$ of occasions between age 14 and age 16 ( $M=4.46$ occasions among those who reported any).

Adulthood.: Binge drinking in the past month was assessed at ages 21, 24, 27, 30, 33, and 39 , with a single item that asked participants to estimate the total number of occasions where they had 5 or more drinks at once in the past month (i.e. "How many times did you drink alcohol in the past month?"). Participants reported at least a single occasion of binge drinking on $25 \%$ of occasions between age 21 and 39 ( $M=4.06$ occasions among those who reported any).

\section{AUD Symptoms}

Adolescence.: AUD symptoms were not measured during adolescence.

Adulthood.: Self-report of 11 DSM-IV symptoms of alcohol abuse and dependence (American Psychiatric Association, 1994) was obtained at ages 21, 24, 27, 30, 33, and 39 using the DIS, as described above. We computed a single score for AUD symptoms by counting the number of criteria met in the past year at each adult wave $(a=.80-.85)$. 


\section{Covariates}

Externalizing: Adolescence.: For all models, we controlled for parent report of adolescent externalizing symptoms from ages $14-16$, as they had been previously shown to be associated with depression and substance use (Kosterman et al., 2010) and, when accounted for, to reduce the association of adolescent internalizing with adult AUD to nonsignificance (Farmer et al., 2016). We used 15 items assessing hyperactivity, oppositionality and conduct problems from the Child Behavior Checklist (Achenbach \& Edlebrock, 1993). Items included "Can't concentrate", "Is impulsive", "Argues a lot", and "Gets in fights". Response options ranged from $1=$ Not at all true to $3=$ Often true. Similar to the computation of the adolescent internalizing variable, we computed a grand-mean centered average of all items across ages $14-16$ ( $a=.85-87$ at each age), reflecting the average level of externalizing symptoms across those ages (reliability $=.83$ ). We used parent report of externalizing symptoms because the adolescent self-report surveys measured a narrower range of externalizing behaviors relative to the parent surveys ${ }^{1}$. We chose to focus on the parent report of externalizing in adolescence because the additional parent-report items formed a much broader construct that included impulsivity, attention problems, and conduct problems, while the adolescent self-report measure focused exclusively on three conduct problems (fighting, stealing, and vandalism). Parent and child report of externalizing were moderately correlated across time $(r=.33-.44, p<.001)$.

Externalizing: Adulthood.: For models predicting adult alcohol outcomes, we also controlled for externalizing in adulthood, using a measure of adult criminality used in prior research (Hawkins et al., 2008). At all adult waves, participants reported on the number of times in the past year that they engaged in various criminal, aggressive and delinquent behaviors, such as major or minor theft, major and minor traffic violations, giving false information, use of a weapon, assault, threats, and justice system involvement (arrests, time spent in jail, etc.). One item measured selling illicit drugs, but use of drugs was not included. Because these reports were highly skewed (with most observations at or near zero), we transformed this variable as follows: We first winsorized the maximum count of each past year behavior to 5 (the $90^{\text {th }}$ to $98^{\text {th }}$ percentile across variables), then standardized each variable within year and computed a mean of all variables across all waves. This variable was correlated $r=.94$ with a variable that was winsorized at 365 , and $r=.63$ with the mean of the non-winsorized variables that included some extreme outliers.

Parent-report of externalizing in adolescence and self-report of externalizing in adulthood were correlated at $r=.30$. Self-report of externalizing in adulthood was weakly correlated with adult trait internalizing at $r=.15$, but not correlated with adolescent internalizing, $r=$ -.03 .

\footnotetext{
${ }^{1}$ We re-analyzed all the models using child self-report of externalizing. For all outcomes, the effects of child vs. parent report of externalizing symptoms was similar in both magnitude, direction and significance in all cases but two. There was stronger continuity of self-reported adolescent and adult externalizing symptoms $(\beta=.55, p<.001)$ than we observed between parent report of adolescent symptoms and adult self-report. Predicting the level of adult alcohol disorder symptoms, the effect of child self-report of externalizing symptoms was positive and significant $(\mathrm{RR}=1.73, p<.01)$, but the effects of parent report were not significant. In no case did the main effects of adult trait internalizing or externalizing change in magnitude, sign, or significance. This suggests that the inferences in the present paper are robust to the choice of reporter of child externalizing symptoms.
} 
Demographic covariates.: For all models, we controlled for race, sex, and early childhood poverty (because the sample was drawn from a higher-risk population). We also controlled for intervention status, comparing the no-treatment control (0) to all other treatment conditions (1). Early childhood poverty was indicated by eligibility for the school free lunch program in ages $10-13$ (52\% of the current sample). Race was coded as White $=1$, NonWhite $=0$, while sex was coded as Male $=1$, Female $=0$. For all adult alcohol outcomes, we controlled for adolescent alcohol use.

\section{Analytic Strategy}

Model Building - Because alcohol outcomes were modeled as a repeated measure over time, we used generalized estimation equations (GEE; Ballinger, 2004; Zeger et al., 1988). GEE was well suited as an analytic approach because a) we did not have specific interest in or hypotheses about random effects (McNeish et al., 2016), b) GEE estimates parameters with the available Level 1 data (i.e. repeated observations of individuals across age), and c) GEE does not require all Level 2 observations (i.e., participants) to have identical or balanced observations at Level 1 (Raudenbush \& Bryk, 2002). To estimate GEEs, we analyzed each alcohol outcome in a general linear model with the appropriate link function (i.e. hurdle negative binomial or logistic models, see details below). Then, we used a custom sandwich estimator with an unstructured correlation matrix to correct model parameter estimates and standard errors for clustering (David Hu, ersonal communication).

All models were estimated using the R packages stats and pscl(Jackman et al., 2015). Though our alcohol outcomes were count-distributed, we had no a priori justification for determining the true data-generating process for each outcome (e.g., zero-inflated negative binominal vs. hurdle negative binomial). For all model comparisons, we selected models by comparing likelihood based indicators to choose the best-fitting distribution for each outcome (e.g. AIC, BIC, and the Vuong test; Vuong, 1989) among a suite of various count distributions (normal, logistic, Poisson, negative binomial, and zero-inflated or hurdle count distributions). This approach allowed us to make an informed decision as to which count distribution best reflected the true response generation process for each of these variables given the data. Our results indicated that alcohol use (\# of past month drinks) and AUD symptoms were best characterized as hurdle negative binomial count distributions, which separately modeled the probability of reporting a non-zero level of outcome (such as any internalizing or AUD symptoms) from the level of the outcome. Count models for binge drinking during both adolescence and adulthood were characterized by inflated parameter estimates and standard errors, a sign of model misspecification. Thus, throughout the analyses, we treated binge drinking as binary $(0=$ no past month binge drinking, $1=$ any past month binge drinking), and estimated a generalized linear model with a logit link function (i.e. logistic regression) with the stats package (R Core Team, 2016). Trait internalizing in adolescence and adulthood were normally distributed, so we estimated a generalized linear model with an identity (i.e., Gaussian) link function in the stats package (R Core Team, 2016). Because it is important to accurately model change over time, we compared linear, quadratic, and piecewise functions of time for adult alcohol outcomes as part of the model building process. In GEE, predictors of individual differences in change over time were entered as moderators of time; thus, we tested whether change over time was moderated by 
all variables in the model, using model fit to judge the significance of these effects. We only included interactions with time that improved model fit by a large amount $(\mathrm{BIC}>10)$ to avoid model overfitting.

Moderation by Sex-For all models, we tested whether interactions of sex with the main effects improved fit. All variables involved in interactions were centered at their respective means to facilitate the interpretation of each coefficient. For all alcohol outcomes, we relied on improvements in model fit based in the Vuong test (Vuong, 1989) to determine whether an interaction was significant, as significance tests of the product term are unreliable in nonlinear models (Ai \& Norton, 2003; Greene, 2010; E. C. Norton et al., 2004; KaracaMandic et al., 2012). We used a criterion of $\mathrm{p}<.01$ to avoid capitalizing on chance interactions.

Interpreting Main Effects-Interpreting main effect coefficients in non-linear models is not as straightforward as the interpretation of linear model coefficients, because the slope (i.e. change in the probability or count of an outcome for a unit change in a predictor) depends on the value of all covariates in a model (King et al., 2000). Even "standardized coefficients" such as odds ratios and risk ratios are difficult to interpret and can be misleading (Sackett et al., 1996). For example, an odds ratio of 2 means that a unit change in a predictor was associated with a doubling of the probability of the outcome, but that could mean a change from $2 \%$ to $4 \%$, or from $25 \%$ to $50 \%$. Thus, to aid interpretation of main effects, we created tables of predicted values of each outcome as a function of interactions at low and high covariate risk using the predict() function in $\mathrm{R}$ (R Core Team). We also created graphical depictions of conditional probabilities and counts of the outcomes, using Monte Carlo simulations of model parameters to estimate confidence intervals at each value of the predictor with the simcf package (Adolph, 2015). We simulated data at specific counterfactual values of the covariates and computed expected values of our outcome based on these estimates to make substantive inferences about these interactions (Greene, 2010; G. King et al., 2000).

Mediation-For adult alcohol outcomes, we tested whether adult trait internalizing mediated the effects of adolescent internalizing symptoms using the product of coefficients method and asymmetric confidence intervals with the package RMediation (Tofighi \& MacKinnon, 2011).

\section{Results}

Table 1 provides descriptive statistics by sex over time.

\section{Associations between Internalizing and Alcohol During Adolescence}

First, we predicted the level and likelihood of alcohol use, and the probability of binge drinking, across ages $14-16$ from internalizing symptoms in two separate models, controlling for adolescent externalizing behaviors and the demographic covariates. Including time in the model predicting alcohol use improved model fit, but produced inflated coefficient estimates and standard errors, a sign of model mis-estimation, so we estimated an intercept only model, which estimates individual differences in the average frequency of 
alcohol use across assessments. Table 2 summarizes these results, and Supplemental Table $1 \mathrm{~S}$ provides full results.

Controlling for the covariates, the frequency and likelihood of alcohol use from ages $14-16$ were strongly associated with average externalizing symptoms across ages $14-16$. There was a time effect for binge drinking: the probability of binge drinking increased with time. Finally, externalizing symptoms were also associated with binge drinking. Internalizing symptoms were unrelated to alcohol use or binge drinking during adolescence in these models.

\section{Stability of Trait Internalizing Within and Across Adolescence and Adulthood}

Next, we predicted trait internalizing in adulthood (i.e. person-level average number of selfreported symptoms of GAD, MDD and social phobia from age 21 - 39) from trait internalizing in adolescence, controlling for externalizing symptoms and adolescent alcohol use and binge drinking (averaged and grand-mean centered across ages $14-16$ ). Table 3 summarizes these results, and Supplemental Table $2 \mathrm{~S}$ provides full results. Above and beyond the effects of the covariates and other predictors, trait internalizing in adolescence was related to trait internalizing in adulthood $(\beta=0.31, p<.001)$. An individual whose internalizing symptoms during adolescence were $1 S D$ above the mean would be expected to have $31 S D$ more internalizing symptoms across adulthood. There was also a significant but small effect of externalizing symptoms $(\beta=0.16, p<.001)$. Adolescent binge drinking, but not adolescent alcohol use, was weakly and positively associated with trait internalizing in adulthood $(\beta=0.11, p=02)$.

By comparison, externalizing symptoms also exhibited moderate continuity with selfreported externalizing in adulthood $(\beta=0.23, p<.001)$, but adolescent internalizing symptoms were unrelated, $p=.88$.

\section{The Association between Adult Alcohol Use and Trait Internalizing}

Table 4 summarizes the association between adult alcohol use and trait internalizing, and Supplemental Table $3 \mathrm{~S}$ provides full results. Including a linear effect of time substantially improved model fit $(\Delta \mathrm{BIC}=-61.26)$, but no higher order polynomials for time improved model fit (all $\Delta \mathrm{BIC}<4$ ), and no interactions with time or sex improved model fit (all $\Delta \mathrm{BIC}$ $<4)$. Thus, all associations with alcohol use reported below reflect associations with individual differences in frequency, but not change over time in frequency, in alcohol use.

First, conditional on the covariates and other predictors, those who reported more frequent alcohol use during adolescence had a higher frequency of alcohol use during adulthood ( $p$ $=.02$ ). Adolescent trait internalizing and externalizing symptoms were unrelated to the likelihood or frequency of alcohol use in adulthood $(p>.13)$.

Neither adult trait internalizing ( $p=0.082)$ nor externalizing $(p=0.754)$ were related to the likelihood of reporting alcohol use in adulthood. On the other hand, both adult trait internalizing $(p=0.011)$ and externalizing $(p=0.000)$ were associated with the frequency of alcohol use in adulthood. 
Table 5 provides the predicted frequency of alcohol use in adulthood at ages 21 and 39, at key levels of adult internalizing and externalizing symptoms. In brief, in early adulthood, trait internalizing was related to modest increases in the frequency of alcohol use. For example, at low levels of externalizing symptoms, a participant $1 S D$ below the mean of trait internalizing who reported drinking was expected to report 2.26 drinking occasions per month, while a participant $1 S D$ above the mean of internalizing symptoms who reported drinking was expected to report around 3 drinking occasions per month. At age 39, the predicted frequencies of drinking were somewhat higher (3.83, 5.00, respectively). For participants $+1 S D$ above the mean on externalizing symptoms, predicted frequencies were even higher at both age 21 and 39 (4.07 and 6.90, respectively). Figure 2 illustrates these effects.

Mediation of Adolescent Internalizing Symptoms-We then tested for an indirect effect of adolescent trait internalizing on adult alcohol use mediated via adult trait internalizing. Although alcohol use was distributed as a hurdle count outcome, meaning there were two possible mediating pathways (via the frequency or likelihood of alcohol use), we only tested mediation effects on the frequency, as the likelihood of use was not associated with trait internalizing.

Results indicated that adult trait internalizing mediated the effects of adolescent internalizing on the frequency of adult alcohol use, $a^{*} b=0.14,95 \% \mathrm{CI}=0.03-0.24$.

\section{The Association between Adult Binge Drinking and Trait Internalizing}

Table 6 reports the findings for binge drinking in adulthood, and Supplemental Table $4 \mathrm{~S}$ provides full results. No variables were associated with change over time (all $\Delta \mathrm{BIC}<4$ ).

Controlling for the covariates, adolescent internalizing symptoms were associated with lower odds of binge drinking across adulthood, $\mathrm{OR}=0.52, p=.004$. Adolescent externalizing symptoms were unrelated to the odds of binge drinking in adulthood $(p=.88)$, while adolescent alcohol use was associated with a higher odds of reporting binge drinking in adulthood ( $\mathrm{OR}=1.07, p=.009$ ). On the other hand, internalizing symptoms during adulthood were associated with a higher likelihood of binge drinking in adulthood (OR = $1.05, p=0.007$ ). Figure 3 illustrates the associations between adult internalizing symptoms and binge drinking.

To compare the effects of adolescent and adult trait internalizing (which were on different scales), we multiplied the raw coefficients by the $S D$ of the predictor, so they represented the predicted log-odds for a $1 S D$ difference in the predictor, and then exponentiated that coefficient. A $1 S D$ increase in adolescent trait internalizing was associated with a $45 \%$ decrease in the odds of reporting binge drinking between ages 21 and 39 , while a $1 S D$ increase in trait internalizing during adulthood was associated with a $54 \%$ increase in the odds of reporting binge drinking.

Mediation of Adolescent Internalizing Symptoms-We found support for an indirect effect of adolescent trait internalizing on binge drinking via adult trait internalizing. Adolescents who reported more internalizing symptoms also reported more internalizing 
symptoms during adulthood, which in turn was associated with a higher probability of binge drinking over time in adulthood.

\section{The Association Between AUD Symptoms and Adult Trait Internalizing}

Next, we predicted AUD symptoms in adulthood from adult trait internalizing, controlling for the covariates, adolescent internalizing and externalizing symptoms and adolescent alcohol use. Including time in the model improved fit, $(\Delta \mathrm{BIC}=190.79)$. Again, however, there was little evidence $(\Delta \mathrm{BIC}<4)$ of interactions with time. Table 7 summarizes this model, and Supplemental Table 5S provides full results.

When all covariates were at zero, the probability of reporting any AUD symptoms was $0.35 \%$, and the mean number of AUD symptoms was 1.25 for those who reported any symptoms. Of the adolescent predictors, adolescent alcohol use ( $\mathrm{OR}=1.08, p<.001)$ was associated with the likelihood of reporting any AUD symptoms. An adolescent whose average alcohol use in adolescence was $1 S D$ higher than the mean would be expected to have $54.37 \%$ greater probability of reporting adult AUD symptoms in adulthood. Adolescent internalizing symptoms were associated with a lower predicted level $(\mathrm{RR}=0.72, p<.05)$ of adult AUD symptoms. An adolescent whose average internalizing in adolescence was $1 S D$ higher than the mean would be expected to report $49.16 \%$ fewer AUD symptoms in adulthood if they reported any at all.

Both adult trait internalizing and externalizing were related to the likelihood and level of AUD symptoms. An individual whose adult trait internalizing was $1 S D$ higher than the mean would be expected to have $59.48 \%$ greater probability of reporting adult AUD symptoms, and would be expected to report $46.80 \%$ more symptoms in adulthood. An individual whose adult externalizing symptoms were $1 S D$ higher than the mean would be expected to have $56.04 \%$ greater probability of reporting adult AUD symptoms, and would be expected to report $27.49 \%$ more symptoms in adulthood. Figures 4 and 5 illustrates these results.

Tables 8 and 9 illustrate the magnitude of these effects. For example, an individual at the mean of all other covariates who was $-1 S D$ on both adult internalizing and externalizing was predicted to have a 59\% probability of reporting any AUD symptoms at age 21, and a $20 \%$ probability of reporting any symptoms at age 39 . Among those who did report AUD symptoms, they reported an average of 1.10 AUD symptoms at age 21 and 1.23 at age 39 . An individual at $+1 S D$ on both internalizing and externalizing had a $78 \%$ probability of reporting any AUD symptoms at age 21 , and a $38 \%$ probability at age 39 . Those who did report AUD symptoms were expected to report 2.47 AUD symptoms at age 21 and 2.75 symptoms at age 39 .

Mediation of Adolescent Trait Internalizing-We then tested for an indirect effect of adolescent trait internalizing on AUD symptoms through adult trait internalizing.

Adolescents who reported higher trait internalizing also reported higher trait internalizing in adulthood, which in turn was associated with the level and likelihood of reporting AUD symptoms in adulthood. In other words, although the direct, partial association of adolescent internalizing symptoms with adult AUD symptoms (controlling for the experience of adult 
internalizing symptoms) was negative, adolescent internalizing symptoms were positively associated with AUD symptoms in adulthood to the degree that they were associated with continued internalizing symptoms in adulthood.

Moderation by Sex-Across all models reported above, we found no evidence for moderation of the effects of trait internalizing by sex (all $p>.05$ ).

\section{Discussion}

In the current study, a trait-like propensity to experience symptoms of internalizing disorders was unrelated to alcohol use and binge drinking in adolescence. However, adolescent trait internalizing exhibited moderate continuity with adult trait internalizing, characterized by symptoms of major depression, generalized anxiety and social phobia. In turn, adult trait internalizing was associated with higher frequency of drinking among those who drank (but no greater propensity to drink than to not drink at all), with a greater likelihood of binge drinking, and with a higher likelihood and levels of AUD symptoms. In this way, adult trait internalizing was a mechanism that partially mediated the association between adolescent trait internalizing and these alcohol outcomes in adulthood. Another way to understand the mediation results is that they demonstrate continuity of trait internalizing across measures and developmental periods. Importantly, these effects were found above and beyond the effects of externalizing symptoms in adolescence and adulthood, and above adolescent alcohol involvement. We did not observe strong evidence that these associations differed across time or sex.

Our results were consistent with theories and prior studies of trait internalizing, which suggest that some individuals exhibit a stable vulnerability to experience emotional distress and fear across the lifespan, which gives rise to trait internalizing and disorders (Kushner et al., 2012). It is particularly notable that we observed moderate rank-order stability in trait internalizing across adolescence and adulthood given the differences across measures in adolescence (where we had relatively more items indicating anxiety vs. depressive symptoms) and adulthood (where we used diagnostic interviews to assess the presence of past-year DSM-IV symptoms of internalizing disorders). It is likely that adolescent and adult measures that cover a more similar range of internalizing symptoms would exhibit even more continuity across development.

The risk posed by trait internalizing for alcohol use, binge drinking and AUDs only emerged in adulthood, and emerged even when controlling for adolescent psychopathology and alcohol involvement. Prior cross-sectional and longitudinal research showed that trait internalizing and AUDs were correlated in adulthood (Jackson \& Sher, 2003; Kushner et al., 2012), but findings in adolescence have been mixed, perhaps in part because the risk represented by trait internalizing only emerges after a period of exposure to and experience with alcohol (Chassin et al., 2016; Colder et al., 2013; Colder et al., 2017; Colder et al., 2018). Indeed, in the current study, we observed no associations between trait internalizing and the likelihood of alcohol use or binge drinking during adolescence, and adolescent trait internalizing was negatively associated with the likelihood of reporting binge drinking or the level of AUD symptoms in adulthood after controlling for adult trait internalizing. Variance 
unique to adolescent trait internalizing may reflect disorder-specific variance, or it may reflect differences in how we measured adolescent vs. adult internalizing (i.e. CBCL vs. a diagnostic interview). It may also be that internalizing symptoms that arise from transient factors (such as environmental stressors) are protective against the development of problem alcohol use.

On the other hand, trait internalizing observed between ages 21 and 39 was associated with higher levels of alcohol use, a higher probability of binge drinking, and a higher probability and level of AUD symptoms. Moreover, the effects of early trait internalizing on alcohol outcomes were mediated via adult trait internalizing, even after controlling for adolescent and adult externalizing, and adolescent alcohol involvement. In other words, the variance common to our measures of internalizing that was also stable over time predicted problematic alcohol involvement in adulthood. This suggests that trait internalizing may be unrelated to alcohol use during adolescence but may identify individuals who are at risk for developing problematic patterns of alcohol involvement after sufficient exposure to alcohol. Upon exposure to alcohol use, individuals high on trait internalizing may be especially prone to be rewarded and reinforced for alcohol use because they are more sensitive to the affectdampening properties of alcohol. Or, to state it another way, trait internalizing may lead to a negatively reinforced pattern of negative-affect motivated alcohol use (i.e., where alcohol is increasingly used to relieve negative emotions). However, to understand the processes by which trait internalizing leads to problematic patterns of substance use, future studies are needed which can characterize between-person differences in the within-person associations between negative affect and alcohol use. For example, ecological momentary assessment (EMA) studies can measure within-person processes (Trull \& Ebner-Priemer, 2014), and burst EMA studies, which administer multiple EMAs over a period of time (Rast et al., 2012) could be used to observe how the negative affect-alcohol use relation changes as a function of time across levels of trait internalizing.

It will also be important to understand when the association between trait internalizing and alcohol outcomes is solidified. Although we could distinguish between adulthood and adolescence in the present study, we could not clearly identify a "tipping point" at which the association became significant. Much like adolescence is a time of experimentation with alcohol use, drinking behaviors during early adulthood are also likely to be more unstable than in later adulthood due to transitions in social contexts (e.g. college entry and exit) and legal barriers to drinking that may coincide with alcohol reinforcement experiences strengthening links to trait internalizing. Future research should seek to understand how and when trait internalizing becomes a prominent predictor of alcohol outcomes.

Prior research has indicated that temperament and personality predict substantial variance in the development of alcohol use and disorders (Sher, Grekin \& Williams, 2005). One prior study reported that both trait internalizing and AUDs in adulthood shared variance with trait neuroticism and behavioral undercontrol, among other risk factors (Jackson \& Sher, 2003).

Similarly, Kusher, Tackett and Bagby (2012) showed that neuroticism was highly correlated with trait internalizing in middle childhood. This suggests that the temperamental, environmental and genetic factors such as neuroticism, (Barlow et al., 2014), negative urgency (Cyders \& Smith, 2008), stress exposure (King \& Chassin, 2008; Trim et al., 2007) 
or low serotonergic functioning (which has been linked to internalizing disorders, Carver et al., 2009) may raise the risk for trait internalizing, and in turn problem alcohol use. Understanding the developmental course by which trait internalizing arises from antecedents earlier in development (such as temperament and early environmental factors) could inform preventive intervention and is an important focus of future research.

One recent study found no associations between childhood and adolescent internalizing symptoms and adult onset of AUDs (Farmer et al., 2016), while we found that that adolescent trait internalizing was actually negatively associated with the likelihood of alcohol use, binge drinking, and AUD symptoms in adulthood, after controlling for adult trait internalizing. There are several differences between Farmer and colleagues' study and the current one worth noting. First, in that study, both AUD and internalizing disorders were treated more as discrete events in terms of onset, while in the current study we considered the average propensity to report internalizing or AUD symptoms (or binge drinking) during adulthood and their rate of change over time (similar to Jackson \& Sher, 2003). Specifically, Farmer and colleagues focused on predicting the onset of meeting threshold criteria for AUDs, rather than continuous AUD symptom counts (2016). That study may have had somewhat lower power due to subjects who experienced sub-threshold symptoms, although using diagnostic outcomes makes their findings more readily applicable to clinical settings. On the other hand, the use of symptom counts in the current study likely improved our power to detect effects but may not be as readily translatable into clinical settings.

Prior studies have shown that there are gender differences in both the risk factors for and the levels of alcohol use and disorders (Nolen-Hoeksema, 2004), and even that there may be stronger associations between risk factors and alcohol outcomes for women than men (Foster et al., 2015). Our study found main effects of gender (indicated by biological sex), such that males exhibited higher likelihood and level of alcohol use, and a higher likelihood of binge drinking, but we found no support for moderation of the effects of trait internalizing by gender. Our findings suggest that interventions which target the putative mechanisms of the association of trait internalizing with alcohol outcomes, such as emotion dysregulation and coping motives for alcohol use, may be expected to have similar effects among men and women. That said, one important caution of this finding is that sex did have a strong main effect on alcohol outcomes, in that males reported higher likelihood and levels of alcohol use and a higher level of binge drinking in adulthood. This introduces a model-inherent interaction (McCabe et al., under review), because males have a different probability or level of the outcome than females. Because the slope of every other predictor is conditional on the probability of the outcome, that means that sex may "moderate" those effects, in that the predictor's effect differs for males vs. females (see also Ai \& Norton, 2003). Methods to accurately understand interactions for nonlinear outcomes are only just being developed, and future research should carefully attend to accurately interpreting non-linear models such as binary and count outcomes (McCabe et al., under review).

\section{Limitations}

There are several limitations we should note. First, although we believe that interventions designed to improve emotion regulation or reduce trait internalizing may aid in the 
prevention or treatment of problematic alcohol involvement (e.g. Lammers et al., 2015), because the present analyses were observational, we cannot decisively conclude a direction of effect, and thus cannot provide conclusive evidence for this suggestion. Indeed, these analyses largely attempt to characterize the patterns of alcohol use over time that were associated with trait internalizing, not to disentangle the within-person processes that drives their associations (which are likely bidirectional). Moreover, our diagnostic procedure may have over-inflated the covariance between disorders that share similar symptoms (e.g., sleep problems specific to depression, and sleep problems specific to alcohol use) to the extent that participants did not distinguish whether overlapping symptoms occurred only in the context of one specific disorder. However, the structured clinical interview was designed to assess symptoms in the context of potential episodes of specific disorders (such as sleeping problems specific to depression), so we believe this concern is minimized.

\section{Summary and Future Directions}

Although prior research has examined the association between internalizing symptoms and alcohol outcomes in both adolescence (Colder et al., 2018, 2013, 2017) and adulthood (i.e. Jackson \& Sher, 2003), no studies have examined both in the same sample across such a wide age range, nor used as broad a range of outcomes. Moreover, by taking an approach that explicitly modeled stable individual differences over time, we can be more confident that our findings were not confounded by time-specific variations in internalizing symptoms (Curran \& Bauer, 2011; King et al., 2018). Our findings provide evidence that a propensity to experience symptoms of internalizing disorders is observable across adolescence and adulthood. The risk of trait internalizing for problem alcohol outcomes, however, was limited to adulthood. This suggests that it may be important to understand the precursors of the trait internalizing-alcohol association during adolescence (such as the development of expectancies and motives), but it may be less reasonable to expect a direct effect on alcohol outcomes. Instead, adulthood, in the 20s and 30s, may be the optimal developmental period in which to understand the within-person processes that link trait internalizing with the development of AUDs.

\section{Supplementary Material}

Refer to Web version on PubMed Central for supplementary material.

\section{Acknowledgments}

This research was supported by grants R01DA09679-11, R01DA021426-08, R01DA033956-05, R01DA08093-12, and R01DA047247 from the National Institute on Drug Abuse, and \#21548 from the Robert Wood Johnson Foundation. The content of this paper is solely the responsibility of the authors and does not necessarily represent the official views of the funding agencies. We thank Andrea Hussong and Craig Colder for their helpful comments on earlier versions of this work.

\section{References}

Achenbach TM, \& Edlebrock C (1993). Manual for the child behavior checklist and revised child behavior profile. Burlington: University of Vermont, Department of Psychiatry.

Adolph C (2015). simcf: Counterfactuals and confidence intervals for estimated regression models. R Package. 
Ai C, \& Norton EC (2003). Interaction terms in logit and probit models. Economics Letters, 80(1), 123-129. 10.1016/S0165-1765(03)00032-6

Association, A. P. (1994). Diagnostic and statistical manual of mental disorders, (DSM-IV). Washington DC, APA (4th ed., Vol. 4th Ed.). American Psychiatric Association.

Ballinger GA (2004). Using generalized estimating equations for longitudinal data analysis. Organizational Research Methods, 7(2), 127-150. 10.1177/1094428104263672

Barlow DH, Sauer-Zavala S, Carl JR, Bullis JR, \& Ellard KK (2014). The nature, diagnosis, and treatment of neuroticism: Back to the future. Clinical Psychological Science, 2(3), 344-365. $10.1177 / 2167702613505532$

Blume SB (1991). Sexuality and stigma: The alcoholic woman. Alcohol Research, 15(2), 139.

Carver CS, Johnson SL, \& Joormann J (2009). Two-mode models of self-regulation as a tool for conceptualizing effects of the serotonin system in normal behavior and diverse disorders. Current Directions in Psychological Science, 18(4), 195-199. https://doi.Org/10.1111/ j.1467-8721.2009.01635.x [PubMed: 20161026]

Chassin L, Colder CR, Hussong AM, \& Sher KJ (2016). Substance use and substance use disorders In Developmental psychopathology, 3rd ed. (Vol. 3). Wiley 10.1002/9781119125556.devpsy319

Chassin L, Ritter J, Trim RS, \& King KM (2003). Adolescent substance use disorders. Child Psychopathology (2nd Ed.)., 199-230. Retrieved from http://ovidsp.ovid.com/ovidweb.cgi? $\mathrm{T}=\mathrm{JS} \& \mathrm{PAGE}=$ reference $\& \mathrm{D}=\mathrm{psyc} 4 \&$ NEWS=N\&AN=2003-04378-004

Chassin L, Tetzloff C, \& Hershey M (1985). Self-image and social-image factors in adolescent alcohol use. Journal of Studies on Alcohol, 46(1), 39-47. [PubMed: 3974234]

Cloninger CR (1987). Neurogenetic adaptive mechanisms in alcoholism. Science (New York, N.Y.), 236, 410-416. 10.1126/science.2882604

Colder CR, Frndak S, Lengua LJ, Read JP, Hawk LW, \& Wieczorek WF (2018). Internalizing and externalizing problem behavior: A test of a latent variable interaction predicting a two-part growth model of adolescent substance use. Journal of Abnormal Child Psychology, 46(2), 319-330. 10.1007/s10802-017-0277-6 [PubMed: 28229368]

Colder CR, Scalco M, Trucco EM, Read JP, Lengua LJ, Wieczorek WF, \& Hawk LW (2013). Prospective associations of internalizing and externalizing problems and their co-occurrence with early adolescent substance use. Journal of Abnormal Child Psychology, 41(4), 667-677. 10.1007/ s10802-012-9701-0 [PubMed: 23242624]

Colder CR, Shyhalla K, Frndak S, Read J, Lengua LJ, Hawk L, \& Wieczorek W (2017). The prospective association between internalizing symptoms and adolescent alcohol involvement and the moderating role of age and externalizing symptoms. Alcoholism: Clinical and Experimental Research, 41(12), 2185-2196. 10.1111/acer.13512

Curran PJ, \& Bauer DJ (2011). The disaggregation of within-person and between-person effects in longitudinal models of change. Annual Review of Psychology, 62, 583-619. 10.1146/ annurev.psych.093008.100356

Cyders MA, \& Smith GT (2008). Emotion-based dispositions to rash action: Positive and negative urgency. Psychological Bulletin, 134(6), 807-828. 10.1037/a0013341 [PubMed: 18954158]

Dawson DA, Goldstein RB, Moss HB, Li T-K, \& Grant BF (2010). Gender differences in the relationship of internalizing and externalizing psychopathology to alcohol dependence: Likelihood, expression and course. Drug and Alcohol Dependence, 112(1-2), 9-17. [PubMed: 20558014]

Eaton NR, Krueger RF, \& Oltmanns TF (2011). Aging and the structure and long-term stability of the internalizing spectrum of personality and psychopathology. Psychology and Aging, 26(4), 987993. 10.1037/a0024406 [PubMed: 21728443]

Enders CK, \& Tofighi D (2007). Centering predictor variables in cross-sectional multilevel models: A new look at an old issue. Psychological Methods, 12(2), 121-138. 10.1037/1082-989X.12.2.121 [PubMed: 17563168]

Farmer RF, Gau JM, Seeley JR, Kosty DB, Sher KJ, \& Lewinsohn PM (2016). Internalizing and externalizing disorders as predictors of alcohol use disorder onset during three developmental periods. Drug and Alcohol Dependence, 164, 38-46. 10.1016/j.drugalcdep.2016.04.021 [PubMed: 27141839] 
Fleming CB, Mason WA, Mazza JJ, Abbott RD, \& Catalano RF (2008). Latent growth modeling of the relationship between depressive symptoms and substance use during adolescence. Psychology of Addictive Behaviors, 22(2), 186-197. 10.1037/0893-164X.22.2.186 [PubMed: 18540716]

Foster KT, Hicks BM, Iacono WG, \& McGue M (2015). Gender differences in the structure of risk for alcohol use disorder in adolescence and young adulthood. Psychological Medicine, 45( 14), $3047-$ 3058. [PubMed: 26118496]

Gottfredson NC (2018). A straightforward approach for coping with unreliability of person means when parsing within-person and between-person effects in longitudinal studies. Addictive Behaviors, 94, 156-161. [PubMed: 30287075]

Greene W (2010). Testing hypotheses about interaction terms in nonlinear models. Economics Letters, 107(2), 291-296. 10.1016/j.econlet.2010.02.014

Sher KJ, Grekin ER, \& Williams NA (2005). The development of alcohol use disorders. Annual Review of Clinical Psychology, 1, 493-523.

Griffith JW, Zinbarg RE, Craske MG, Mineka S, Rose RD, Waters AM, \& Sutton JM (2010). Neuroticism as a common dimension in the internalizing disorders. Psychological Medicine, 40(7), 1125-1136. 10.1017/S0033291709991449 [PubMed: 19903363]

Hawkins JD, Catalano RF, Kosterman R, Abbott R, \& Hill KG (1999). Preventing adolescent healthrisk behaviors by strengthening protection during childhood. Archives of Pediatrics \& Adolescent Medicine, 153(3), 226-234. 10.1001/archpedi.153.3.226 [PubMed: 10086398]

Hawkins JD, Catalano R, Morrison D, O’Donnell J, Abbott R, \& Day L (1992). The Seattle Social Development Project: Effects of the first four years on protective factors and problem behaviors In Preventing antisocial behavior: Interventions from birth through adolescence (pp. 139-161). Guilford Press Retrieved from http://www.ssdp-tip.org/SSDP/interventionpapers/Hawkins-1992tx-1st-4-years.pdfl\%5Cnhttp://psycnet.apa.org/psycinfo/1992-98237-007

Hawkins JD, Guo J, Hill KG, Battin-Pearson S, \& Abbott RD (2001). Long-term effects of the Seattle Social Development Intervention on school bonding trajectories. Applied Developmental Science, 5(4), 225-236. 10.1207/S1532480XADS0504_04 [PubMed: 17955057]

Hawkins JD, Kosterman R, Catalano RF, Hill KG, \& Abbott RD (2005). Promoting positive adult functioning through social development intervention in childhood: Long-term effects from the Seattle Social Development Project. Archives of Pediatrics \& Adolescent Medicine, 159(1), 2531. [PubMed: 15630054]

Hawkins JD, Kosterman R, Catalano RF, Hill KG, \& Abbott RD (2008). Effects of social development intervention in childhood 15 years later. Archives of Pediatrics and Adolescent Medicine, 162(12), 1133-1141. 10.1001/archpedi.162.12.1133 [PubMed: 19047540]

Hussong AM, Curran PJ, \& Chassin L (1998). Pathways of risk for accelerated heavy alcohol use among adolescent children of alcoholic parents. Journal of Abnormal Child Psychology, 26(6), 453-466. 10.1023/A:1022699701996 [PubMed: 9915652]

Hussong AM, Ennett ST, Cox MJ, \& Haroon M (2017). A systematic review of the unique prospective association of negative affect symptoms and adolescent substance use controlling for externalizing symptoms. Psychology of Addictive Behaviors, 31(2), 137-147. 10.1037/adb0000247 [PubMed: 28134539]

Hussong AM, Jones DJ, Stein GL, Baucom DH, \& Boeding S (2011). An internalizing pathway to alcohol use and disorder. Psychology of Addictive Behaviors, 25(3), 390-404. 10.1037/a0024519 [PubMed: 21823762]

Jackman S, Tahk A, Zeileis A, Maimone C, \& Fearon J (2015). Package “pscl”. Retrieved from http:// politicalbubbles.us/pscl.pdf

Jackson KM, \& Sher KJ (2003). Alcohol use disorders and psychological distress: A prospective statetrait analysis. Journal of Abnormal Psychology, 112(4), 599-613. 10.1037/0021-843X.112.4.599 [PubMed: 14674872]

Jaffee SR, Moffitt TE, Caspi A, Fombonne E, Poulton R, Martin J, ... al. (2002). Differences in early childhood risk factors for juvenile-onset and adult-onset depression. Archives of General Psychiatry, 59(3), 215 10.1001/archpsyc.59.3.215 [PubMed: 11879158] 
Johnston LD, O’Malley PM, Miech RA, Bachman JG, \& Schulenberg JE (2016). Monitoring the future national survey results on drug use, 1975-2015: Overview, key findings on adolescent drug use. Ann Arbor: Institute for Social Research, the University of Michigan.

Kaplow JB, Curran PJ, Dodge KA, Bierman KL, Coie JD, Greenberg MT, ... Pinderhughes EE (2002). Child, parent, and peer predictors of early-onset substance use: A multisite longitudinal study. Journal of Abnormal Child Psychology, 30(3), 199-216. 10.1023/A:1015183927979 [PubMed: 12041707]

Karaca-Mandic P, Norton EC, \& Dowd B (2012). Interaction terms in nonlinear models. Health Services Research, 47(1 PART 1), 255-274. 10.1111/j.1475-6773.2011.01314.x [PubMed: 22091735]

Kessler RC, Berglund P, Demler O, Jin R, Merikangas KR, \& Walters EE (2005). Lifetime Prevalence and age-of-Onset Distributions of DSM-IV Disorders in the National Comorbidity Survey Replication. Archives of General Psychiatry, 62(6), 593 10.1001/archpsyc.62.6.593 [PubMed: 15939837]

King G, Tomz M, \& Wittenberg J (2000). Making the most of statistical analyses: Improving interpretation and presentation. American Journal of Political Science, 44(2), 347-361. $10.2307 / 2669316$

King KM, \& Chassin L (2008). Adolescent stressors, psychopathology, and young adult substance dependence: A prospective study. Journal of Studies on Alcohol and Drugs, 69(5), 629-638. [PubMed: 18781237]

King KM, Littlefield AK, McCabe CJ, Mills KL, Flournoy J, \& Chassin L (2018). Longitudinal modeling in developmental neuroimaging research: Common challenges, and solutions from developmental psychology. Developmental Cognitive Neuroscience. 10.1016/j.dcn.2017.11.009

King SM, Iacono WG, \& McGue M (2004). Childhood externalizing and internalizing psychopathology in the prediction of early substance use. Addiction, 99(12), 1548-1559. 10.1111/ j.1360-0443.2004.00893.x [PubMed: 15585046]

Klassen AD, \& Wilsnack SC (1986). Sexual experience and drinking among women in a us national survey. Archives of Sexual Behavior, 15(5), 363-392. [PubMed: 3789902]

Koob GF, \& Le Moal M (2008). Addiction and the brain antireward system. Annual Review of Psychology, 59, 29-53. 10.1146/annurev.psych.59.103006.093548

Kosterman R, Hawkins JD, Mason WA, Herrenkohl TF, Lengua LJ, \& McCauley E (2010). Assessment of behavior problems in childhood and adolescence as predictors of early adult depression. Journal of Psychopathology and Behavioral Assessment, 32(1), 118-127. 10.1007/ s10862-009-9138-0

Kosterman R, Hawkins JD, Bailey JA, Catalano RF, Abbott RD, \& Hill KG (2019). Effects of social development intervention in childhood on adult life at ages 30 to 39. Prevention Science. 20 986995. [PubMed: 31152329]

Krueger RF, \& Markon KE (2006). Reinterpreting comorbidity: A model-based approach to understanding and classifying psychopathology. Annual Review of Clinical Psychology, 2(1), 111-133. 10.1146/annurev.clinpsy.2.022305.095213

Kushner MG, Wall MM, Krueger RF, Sher KJ, Maurer E, Thuras P, \& Lee S (2012). Alcohol dependence is related to overall internalizing psychopathology load rather than to particular internalizing disorders: Evidence from a national sample. Alcoholism: Clinical and Experimental Research, 36(2), 325-331. 10.1111/j.1530-0277.2011.01604.x

Lammers J, Goossens F, Conrod P, Engels R, Wiers RW, \& Kleinjan M (2015). Effectiveness of a selective intervention program targeting personality risk factors for alcohol misuse among young adolescents ..., (8), 10.1111/add.12952

Lüdtke O, Marsh HW, Robitzsch A, Trautwein El., Asparouhov T, \& Muthén B (2008). The multilevel latent covariate model: A new, more reliable approach to group-level effects in contextual studies. Psychological Methods, 13(3), 203-229. 10.1037/a0012869 [PubMed: 18778152]

McCabe CJ, Halvorson MA, King KM, Cao X, \& Kim DS (under review). Addressing the misinterpretation of interaction effects in generalized linear models: An example using the Adolescent Brain Cognitive Development (ABCD) Study. 
McCabe CJ, Kim DS, \& King KM (2018). Improving Present Practices in the Visual Display of Interactions. Advances in Methods and Practices in Psychological Science, 1(2), 147-165. $10.1177 / 2515245917746792$

McNeish D, Stapleton LM, \& Silverman RD (2016). On the unnecessary ubiquity of hierarchical linear modeling. Psychological Methods, 55(12), 7250-7257. 10.1037/met0000078

Nolen-Hoeksema S (2004). Gender differences in risk factors and consequences for alcohol use and problems. Clinical Psychology Review, 24(8), 981-1010. 10.1016/j.cpr.2004.08.003 [PubMed: 15533281]

Norton EC, Wang H, \& Ai C (2004). Computing interaction effects and standard errors in logit and probit models. Stata Journal, 4(2), 154-167. 10.1016/S0165-1765(03)00032-6

Rast P, MacDonald SWS, \& Hofer SM (2012). Intensive Measurement Designs for Research on Aging. GeroPsych, 25(2), 45-55. 10.1024/1662-9647/a000054 [PubMed: 24672475]

Raudenbush SW, \& Bryk AS (2002). Hierarchical linear models: Applications and data analysis methods (Vol. 1, p. 485). Los Angeles, CA: Sage.

Sackett DL, Deeks JJ, \& Altman DG (1996). Down with odds ratios! Evidence-Based Medicine, 7(6), 164.

Sher KJ (1991). Children of alcoholics: A critical appraisal of theory and research. Children of Alcoholics: A Critical Appraisal of Theory and Research. Retrieved from http://ovidsp.ovid.com/ ovidweb.cgi?T=JS\&PAGE=reference $\& D=$ psyc3 \&NEWS=N\&AN=1992-97176-000

Sihvola E, Rose RJ, Dick DM, Pulkkinen L, Marttunen M, \& Kaprio J (2008). Early-onset depressive disorders predict the use of addictive substances in adolescence: A prospective study of adolescent Finnish twins. Addiction, 103(12), 2045-2053. 10.1111/j.1360-0443.2008.02363.x [PubMed: 18855807]

Smith GT, \& Cyders MA (2016). Integrating affect and impulsivity: The role of positive and negative urgency in substance use risk. Drug and Alcohol Dependence, 163, S3-S12. 10.1016/ j.drugalcdep.2015.08.038 [PubMed: 27306729]

Team, R. D. C. (2016). R: A language and environment for statistical computing. R Foundation for Statistical Computing Vienna Austria, 0, ISBN 3-900051-07-00. 10.1038/sj.hdy.6800737

Tofighi D, \& MacKinnon DP (2011). RMediation: An r package for mediation analysis confidence intervals. Behavior Research Methods, 43(3), 692-700. 10.3758/s13428-011-0076-x [PubMed: 21487904]

Trim RS, Meehan BT, King KM, \& Chassin L (2007). The relation between adolescent substance use and young adult internalizing symptoms: Findings from a high-risk longitudinal sample. Psychology of Addictive Behaviors, 21(1), 97-107. 10.1037/0893-164X.21.T97 [PubMed: 17385959]

Trull TJ, \& Ebner-Priemer U (2014). The Role of Ambulatory Assessment in Psychological Science. Current Directions in Psychological Science, 23(6), 466-470. 10.1177/0963721414550706 [PubMed: 25530686]

Vuong QH (1989). Likelihood ratio tests for model selection and non-nested hypotheses. Econometrica, 57(2), 307 10.2307/1912557

Wang FL, \& Chassin L (2017). Negative urgency mediates the relation between genetically influenced serotonin functioning and alcohol problems. Clinical Psychological Science, 216770261773381 $10.1177 / 2167702617733817$

Wills TA, \& Filer M (1996). Stress-coping model of adolescent substance use. Advances in Clinical Child Psychology, 18, 91-132.

Zeger SL, Liang KY, \& Albert PS (1988). Models for longitudinal data: A generalized estimating equation approach. Biometrics, 44(4), 1049-1060. 10.2307/2531734 [PubMed: 3233245] 


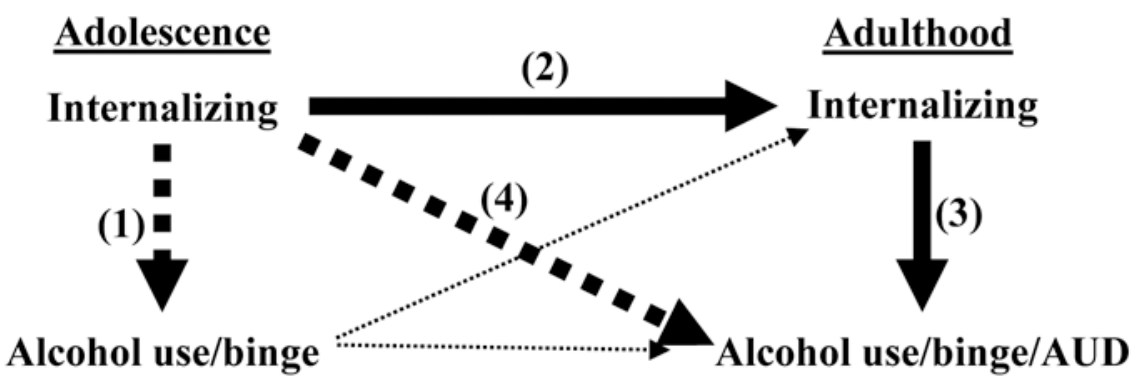

Figure 1.

A heuristic model of hypothesis tests in the current study. 


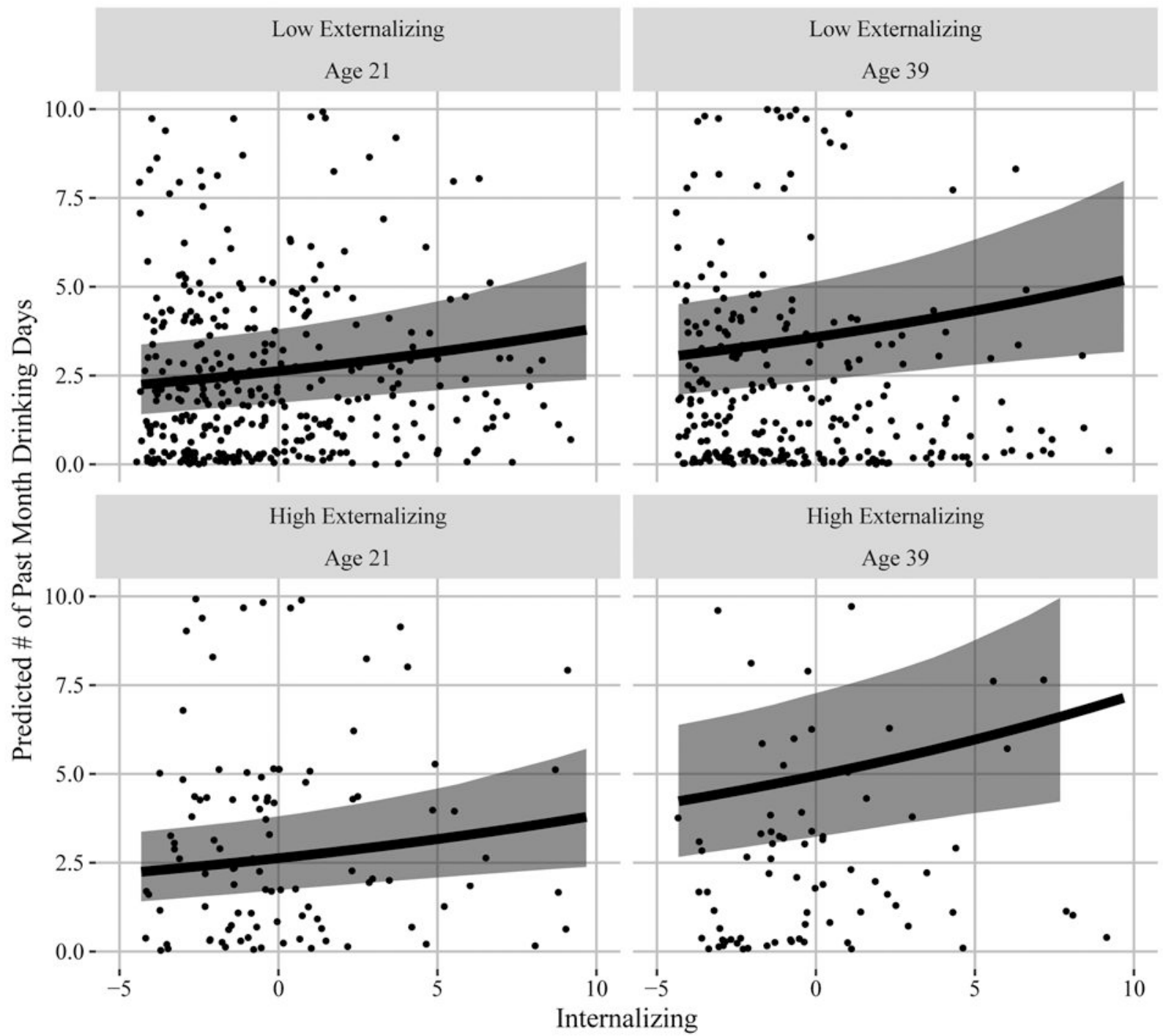

Figure 2.

The predicted association between adult internalizing symptoms and the level of alcohol use, plotted across levels of adult externalizing and age. Confidence intervals were simulated using the simcf package (Adolph, 2015). 


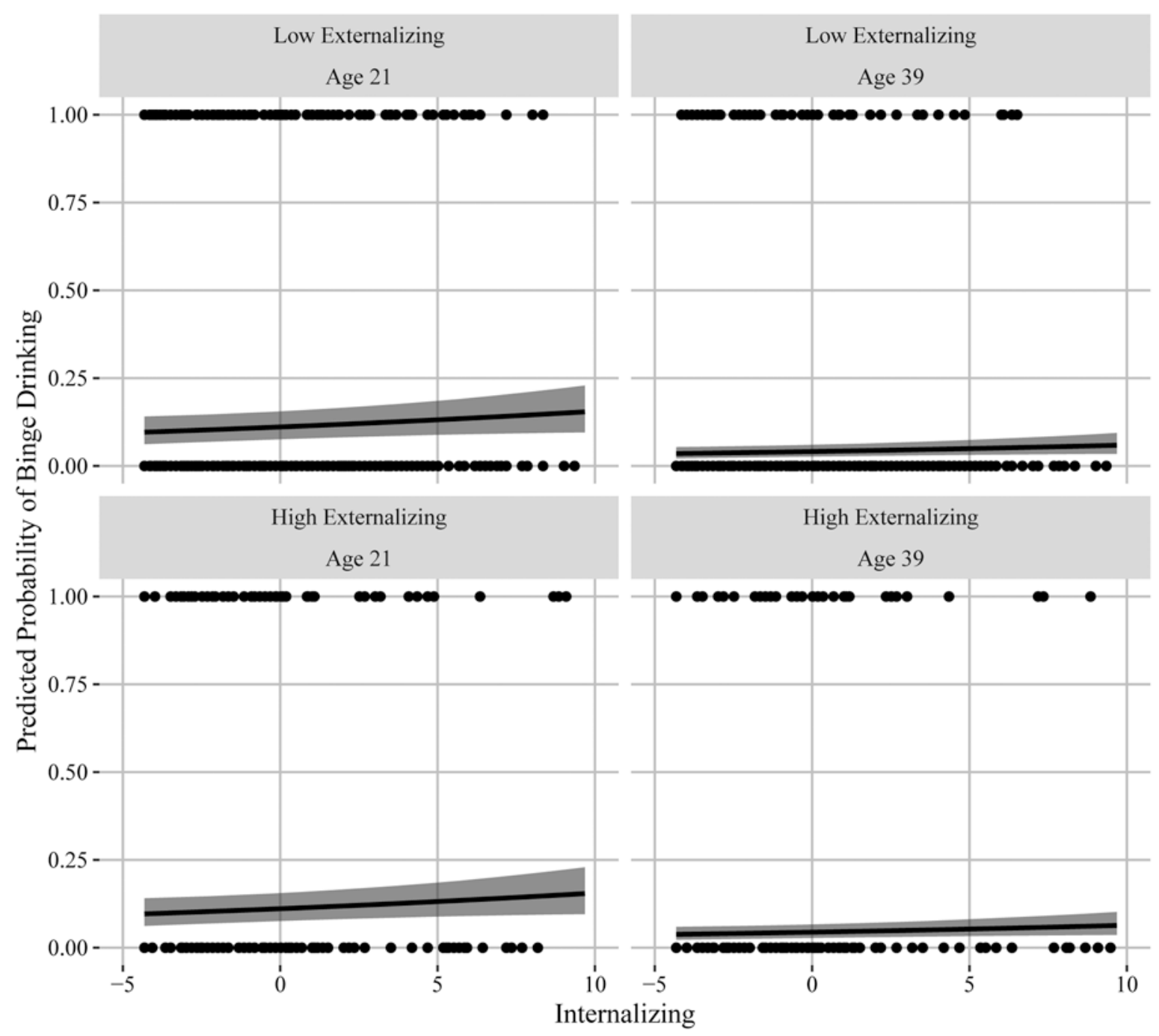

Figure 3.

The predicted association between adult internalizing symptoms and the probability of binge drinking, plotted across levels of adult externalizing and age. Confidence intervals were simulated using the simcf package (Adolph, 2015). 


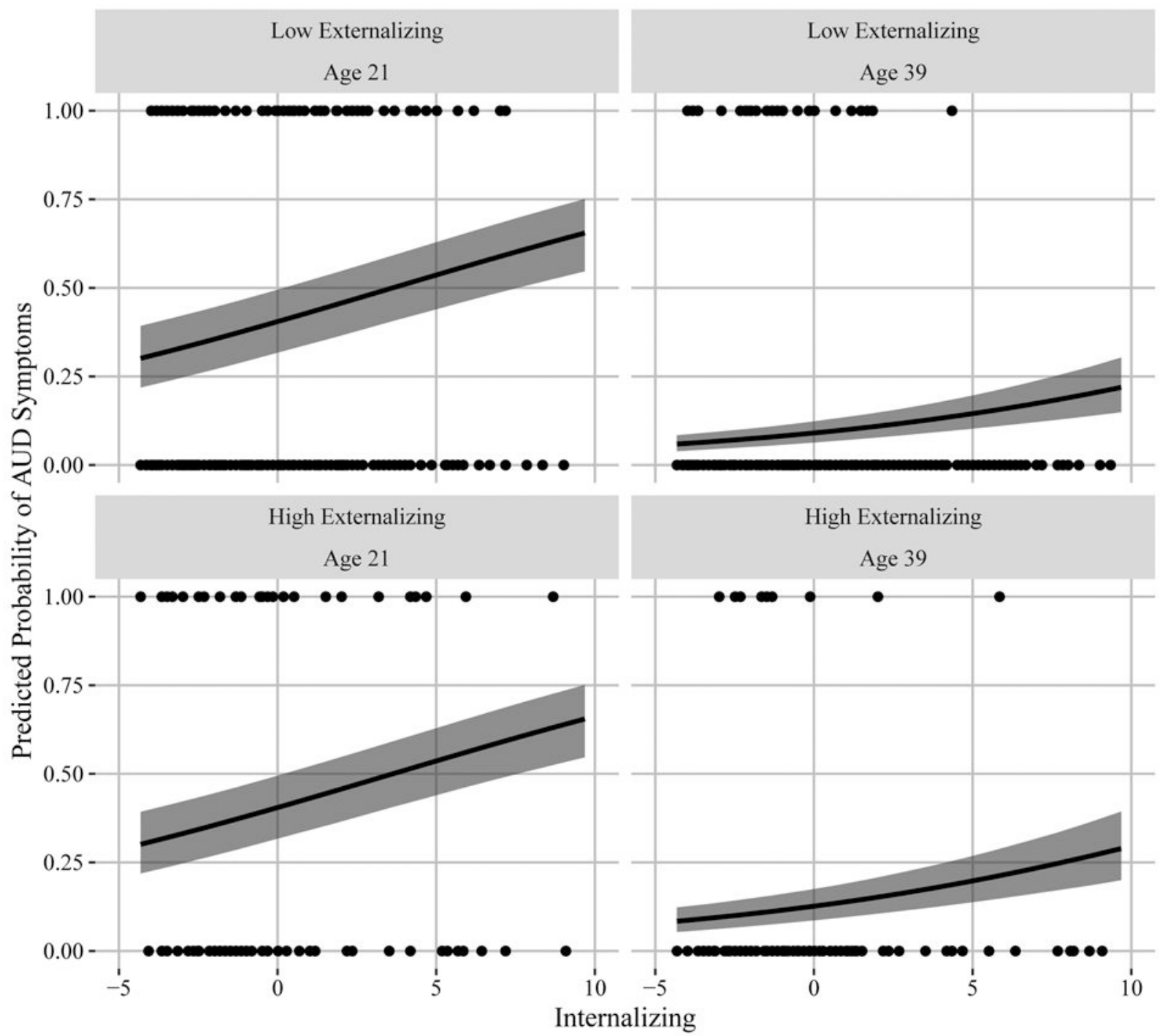

Figure 4.

The predicted association between adult internalizing symptoms and the probability of AUD Symptoms, plotted across levels of adult externalizing and age. Confidence intervals were simulated using the simcf package (Adolph, 2015). 


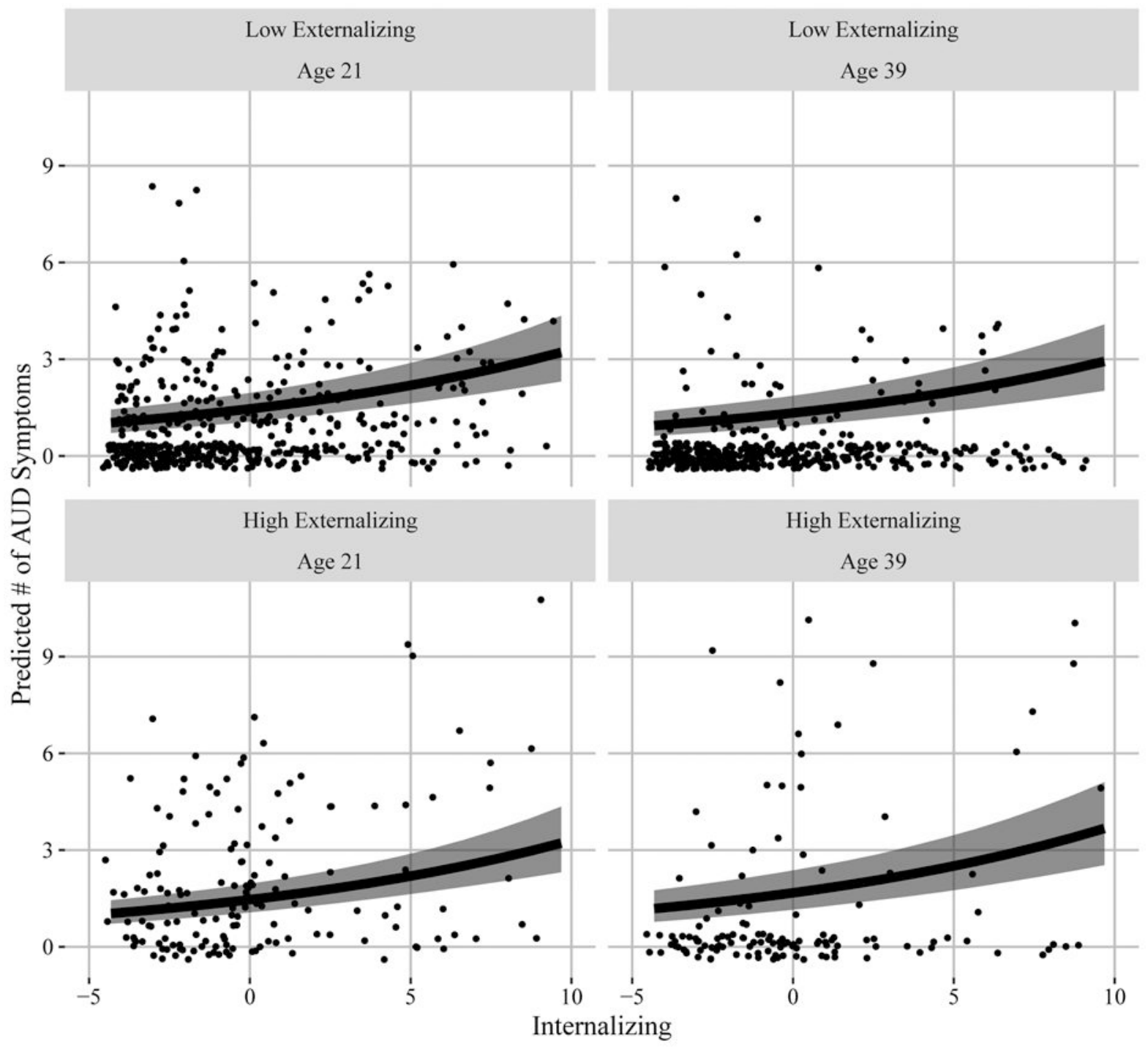

Figure 5.

The predicted association between adult internalizing symptoms and the \# of AUD Symptoms, plotted across levels of adult externalizing and age. Confidence intervals were simulated using the simcf package (Adolph, 2015). 


\section{\.}

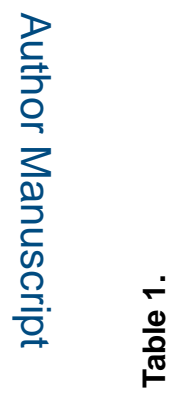

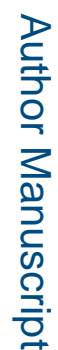

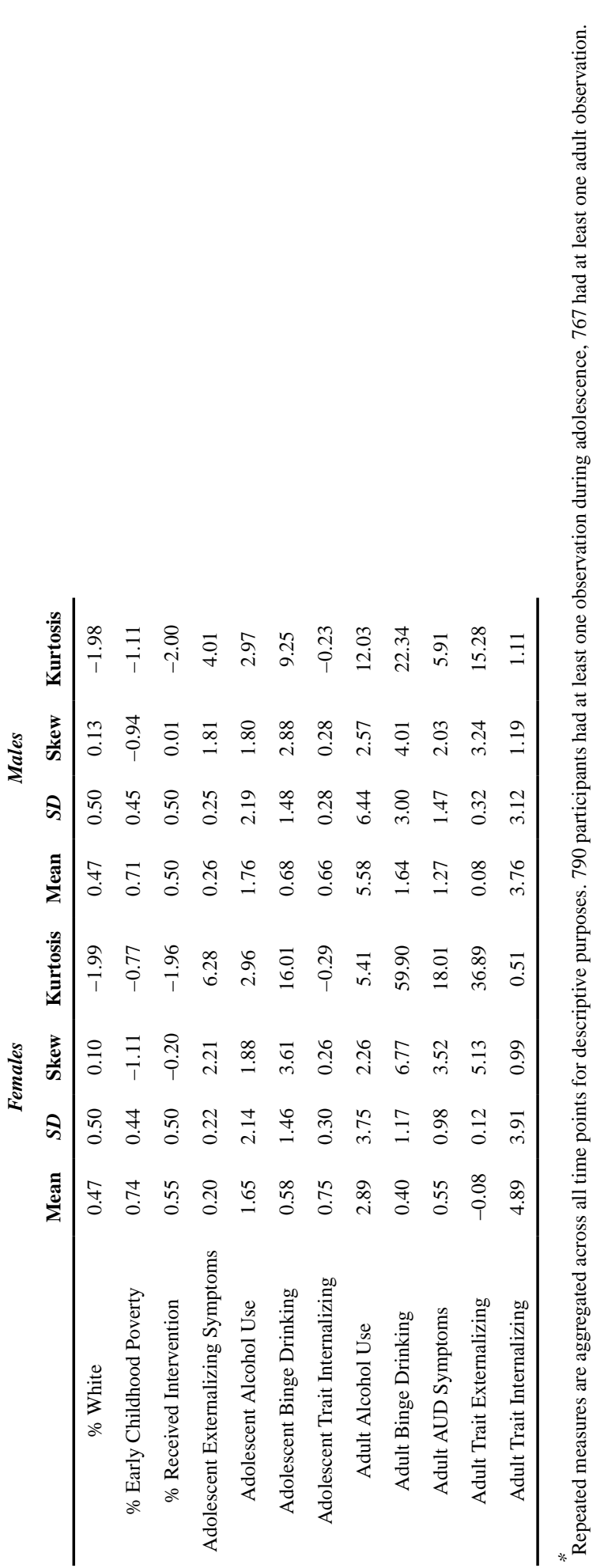

Psychol Addict Behav. Author manuscript; available in PMC 2021 November 01. 
Table 2.

Predicting Adolescent Alcohol Outcomes

\begin{tabular}{cccccc} 
& Estimate & S.E. & p & RR/OR & $\mathbf{9 5 \%}$ CI \\
\hline Alcohol Use Level & & & & & \\
Adolescent Internalizing & -0.22 & 0.2 & 0.266 & 0.80 & $(0.54-1.19)$ \\
Adolescent Externalizing & 1.60 & 0.4 & 0.000 & $\mathbf{4 . 9 7}$ & $(2.27-10.86)$ \\
Alcohol Use Likelihood & & & & & \\
Adolescent Internalizing & -0.02 & 0.12 & 0.875 & 0.98 & $(0.78-1.24)$ \\
Adolescent Externalizing & 0.90 & 0.18 & 0.000 & $\mathbf{2 . 4 6}$ & $(1.72-3.52)$ \\
Binge Use Likelihood & & & & & \\
Time & 0.41 & 0.07 & 0.000 & $\mathbf{1 . 5 1}$ & $(1.31-1.74)$ \\
Adolescent Internalizing & -0.25 & 0.27 & 0.346 & 0.78 & $(0.46-1.31)$ \\
Adolescent Externalizing & 1.90 & 0.29 & 0.000 & $\mathbf{6 . 6 8}$ & $(3.82-11.69)$ \\
\hline
\end{tabular}

Note. Bolded effects are significant at $p<.05$. All effects control for covariates (treatment assignment, childhood poverty, ethnicity); those effects are not displayed for parsimony (See Table $1 \mathrm{~S}$ ). $\mathrm{RR} / \mathrm{OR}=$ Risk ratio/odds ratio, $95 \% \mathrm{CI}=95 \%$ confidence interval 
Table 3.

Predicting Adult Trait Internalizing

\begin{tabular}{cccccc} 
& b & S.E. & $\mathbf{p}$ & Beta & $\mathbf{9 5 \%}$ CI \\
\hline Intercept & 0.69 & & & & \\
Adolescent Internalizing & 3.68 & 0.41 & 0.000 & $\mathbf{0 . 3 0}$ & $(2.88-4.48)$ \\
Adolescent Externalizing & 2.34 & 0.52 & 0.000 & $\mathbf{0 . 1 6}$ & $(1.31-3.36)$ \\
Adolescent Alcohol Use & -0.02 & 0.08 & 0.77 & -0.01 & $(-0.17-0.13)$ \\
Adolescent Binge Drinking & 1.57 & 0.65 & 0.015 & $\mathbf{0 . 1 1}$ & $(0.30-2.85)$ \\
\hline
\end{tabular}

Note. Bolded effects are significant at $p<.05$. All effects control for covariates (treatment assignment, childhood poverty, ethnicity); those effects are not displayed for parsimony (See Table 2S). 
Table 4.

Predicting Adult Alcohol Use

\begin{tabular}{cccccc} 
Level Model & Estimate & S.E. & P & RR/OR & 95\% CI \\
\hline Intercept & 0.74 & & & & \\
Time & 0.11 & 0.01 & 0.000 & $\mathbf{1 . 1 1}$ & $(1.08-1.14)$ \\
Adolescent Internalizing & -0.16 & 0.17 & 0.354 & 0.85 & $(0.61-1.19)$ \\
Adolescent Externalizing & -0.01 & 0.18 & 0.955 & 0.99 & $(0.70-1.41)$ \\
Adolescent Alcohol Use & 0.02 & 0.02 & 0.299 & 1.02 & $(0.98-1.07)$ \\
Adult Externalizing & 0.82 & 0.23 & 0.000 & $\mathbf{2 . 2 8}$ & $(1.45-3.58)$ \\
Adult Trait Internalizing & 0.04 & 0.01 & 0.011 & $\mathbf{1 . 0 4}$ & $(1.01-1.07)$ \\
& Indirect Effect & & $\mathbf{9 5 \%}$ CI \\
\hline Adolescent Internalizing via & & $\mathbf{0 . 1 4}$ & & $(0.03-0.24)$ \\
Adult Internalizing & & & & & \\
Likelihood Model & Estimate & S.E. & $\mathbf{P}$ & $\mathbf{R R / O R}$ & $\mathbf{9 5 \%}$ CI \\
\hline Intercept & 0.51 & & & & \\
Time & -0.06 & 0.02 & 0.000 & 0.94 & $(0.91-0.97)$ \\
Adolescent Internalizing & -0.31 & 0.21 & 0.139 & 0.73 & $(0.49-1.11)$ \\
Adolescent Externalizing & -0.25 & 0.24 & 0.310 & 0.78 & $(0.49-1.26)$ \\
Adolescent Alcohol Use & 0.07 & 0.03 & 0.023 & $\mathbf{1 . 0 7}$ & $(1.01-1.13)$ \\
Adult Externalizing & -0.08 & 0.24 & 0.754 & 0.93 & $(0.58-1.48)$ \\
Adult Trait Internalizing & 0.03 & 0.02 & 0.082 & 1.03 & $(1.00-1.07)$ \\
Negative Binomial Dispersion & 0.452 & - & - & - & - \\
\hline
\end{tabular}

Note. Bolded effects are significant at $p<.05$. All effects control for covariates (treatment assignment, childhood poverty, and ethnicity); those effects are not displayed for parsimony (See Table $3 \mathrm{~S}$ ). $\mathrm{RR} / \mathrm{OR}=$ Risk ratio/odds ratio, $95 \% \mathrm{CI}=95 \%$ confidence interval 
Table 5.

Predicted Adult Alcohol Use (\# of episodes per month) by Age and Level of Adult Externalizing Symptoms

Age 21

Externalizing Symptoms Level

Trait Internalizing Level

$-1 S D$

$+1 S D$

$+2 S D$

$-1 S D$

2.26

3.12

3.83

Mean

2.58

3.56

4.38

$+1 S D$

$\begin{array}{lll}2.95 & 4.07 & 5.00\end{array}$

$+2 S D$

3.37

4.65

5.72

Age 39

Externalizing Symptoms Level

\begin{tabular}{cccc} 
Trait Internalizing Level & $\mathbf{- 1} \boldsymbol{S D}$ & $\mathbf{+ 1} \boldsymbol{S D}$ & $\mathbf{+ 2} \boldsymbol{S D}$ \\
\hline$-1 S D$ & 3.83 & 5.28 & 6.49 \\
Mean & 4.38 & 6.04 & 7.42 \\
$+1 S D$ & 5.00 & 6.90 & 8.48 \\
$+2 S D$ & 5.72 & 7.88 & 9.69 \\
\hline
\end{tabular}

Note. All other covariates held at the sample mean. Low $=-1 / 2 S D$, High $=+1 S D$, Very High $=+2 S D$. 
Table 6.

Predicting Adult Binge Drinking

\begin{tabular}{llllll} 
& Estimate & S.E. & $\boldsymbol{p}$ & OR & OR 95\% CI \\
\hline Intercept & -1.74 & & & & \\
Time & -0.10 & 0.02 & 0.000 & $\mathbf{0 . 9 0}$ & $(0.87-0.93)$ \\
Adolescent Internalizing & -0.66 & 0.23 & 0.004 & $\mathbf{0 . 5 2}$ & $(0.33-0.81)$ \\
Adolescent Externalizing & -0.04 & 0.27 & 0.876 & 0.96 & $(0.57-1.62)$ \\
Adolescent Alcohol Use & 0.07 & 0.03 & 0.009 & $\mathbf{1 . 0 7}$ & $(1.02-1.13)$ \\
Adult Externalizing & 0.40 & 0.24 & 0.093 & 1.50 & $(0.94-2.40)$ \\
Adult Trait Internalizing & 0.05 & 0.02 & 0.007 & $\mathbf{1 . 0 5}$ & $(1.01-1.10)$ \\
& Indirect Effect & $\mathbf{9 5 \%}$ CI & & & \\
Mediated Effect & $\mathbf{0 . 2 0}$ & $(0.01-0.38)$ & & &
\end{tabular}

Note. Bolded effects are significant at $p<.05$. All effects control for covariates (treatment assignment, childhood poverty, and ethnicity); those effects are not displayed for parsimony (See Table $4 \mathrm{~S}$ ). OR = odds ratio, 95\% CI = 95\% confidence interval. 
Table 7.

Predicting Adult AUD Symptoms

\begin{tabular}{cccccc} 
Level Model & Estimate & S.E. & $\boldsymbol{p}$ & $\mathbf{R R}$ & $\mathbf{9 5 \%}$ CI \\
\hline Intercept & 0.22 & & & & \\
Time & 0.02 & 0.02 & 0.232 & 1.02 & $(0.99-1.06)$ \\
Adolescent Internalizing & -0.33 & 0.16 & 0.037 & $\mathbf{0 . 7 2}$ & $(0.53-0.98)$ \\
Adolescent Externalizing & 0.01 & 0.16 & 0.970 & 1.01 & $(0.73-1.38)$ \\
Adolescent Alcohol Use & 0.01 & 0.02 & 0.750 & 1.01 & $(0.97-1.04)$ \\
Adult Externalizing & 0.58 & 0.14 & 0.000 & $\mathbf{1 . 7 8}$ & $(1.35-2.34)$ \\
Adult Trait Internalizing & 0.08 & 0.01 & 0.000 & $\mathbf{1 . 0 8}$ & $(1.06-1.11)$ \\
& Indirect Effect & & & $\mathbf{9 5 \%}$ CI \\
\hline Adolescent Internalizing via & & 0.30 & & $(0.21-0.39)$ \\
Adult Internalizing & & & & & \\
Likelihood Model & Estimate & S.E. & $\boldsymbol{p}$ & $\mathbf{O R}$ & $\mathbf{9 5 \%}$ CI \\
\hline Intercept & -0.63 & & & & \\
Time & -0.26 & 0.02 & 0.000 & $\mathbf{0 . 7 7}$ & $(0.74-0.80)$ \\
Adolescent Internalizing & -0.30 & 0.20 & 0.143 & 0.74 & $(0.50-1.11)$ \\
Adolescent Externalizing & 0.26 & 0.24 & 0.284 & 1.30 & $(0.81-2.09)$ \\
Adolescent Alcohol Use & 0.08 & 0.03 & 0.001 & $\mathbf{1 . 0 8}$ & $(1.03-1.14)$ \\
Adult Externalizing & 0.96 & 0.25 & 0.000 & $\mathbf{2 . 6 0}$ & $(1.59-4.25)$ \\
Adult Trait Internalizing & 0.11 & 0.02 & 0.000 & $\mathbf{1 . 1 1}$ & $(1.08-1.15)$ \\
Negative Binomial & 1.996 & - & - & - & - \\
Dispersion & & & & & \\
\hline
\end{tabular}

Note. Bolded coefficients are significant, $p<.05$. All effects control for covariates (treatment assignment, childhood poverty, and ethnicity); those effects are not displayed for parsimony (See Table $5 \mathrm{~S}$ ). $\mathrm{RR} / \mathrm{OR}=$ risk ratio/odds ratio, $95 \% \mathrm{CI}=95 \%$ confidence interval. 
Table 8.

Predicted probability of AUD symptoms at Age 21 and 39 for different levels of trait internalizing and adult externalizing

\begin{tabular}{cccc} 
Age 21 & \multicolumn{3}{c}{ Externalizing Symptoms Level } \\
Trait Internalizing Level & $\mathbf{- 1}$ SD & $\mathbf{+ 1} \mathbf{S D}$ & $\mathbf{+ 2 S \boldsymbol { D }}$ \\
\hline$-1 S D$ & 0.59 & 0.67 & 0.71 \\
Mean & 0.65 & 0.73 & 0.77 \\
$+1 S D$ & 0.71 & 0.78 & 0.82 \\
+ +2 SD & 0.77 & 0.83 & 0.86 \\
Age 39 & Externalizing Symptoms Level \\
Trait Internalizing Level & $\mathbf{- 1}$ SD & $\mathbf{+ 1} \boldsymbol{S D}$ & $\mathbf{+ 2 S \boldsymbol { D }}$ \\
\hline$-1 S D$ & 0.20 & 0.25 & 0.29 \\
Mean & 0.25 & 0.31 & 0.35 \\
$+1 S D$ & 0.31 & 0.38 & 0.43 \\
$+2 S D$ & 0.37 & 0.45 & 0.51 \\
\hline
\end{tabular}

Note. All other covariates held at the sample mean. Low $=-1 / 2 S D$, High $=+1 S D$, Very High $=+2 S D$. 
Table 9.

Predicted level of AUD symptoms at Age 21 and 39 for different levels of trait internalizing and adult externalizing

\begin{tabular}{cccc} 
Age 21 & \multicolumn{3}{c}{ Externalizing Symptoms Level } \\
Trait Internalizing Level & $\mathbf{- 1} \mathbf{S D}$ & $\mathbf{+ 1} \mathbf{S D}$ & $\mathbf{+ 2 S \boldsymbol { D }}$ \\
\hline$-1 S D$ & 1.10 & 1.38 & 1.59 \\
Mean & 1.47 & 1.85 & 2.13 \\
$+1 S D$ & 1.97 & 2.47 & 2.85 \\
$+2 S D$ & 2.64 & 3.30 & 3.81 \\
Age 39 & Externalizing Symptoms Level \\
Trait Internalizing Level & $\mathbf{- 1} \boldsymbol{S D}$ & $\mathbf{+ 1}$ SD & $\mathbf{+ 2 S \boldsymbol { D }}$ \\
\hline$-1 S D$ & 1.23 & 1.54 & 1.78 \\
Mean & 1.64 & 2.06 & 2.37 \\
$+1 S D$ & 2.20 & 2.75 & 3.17 \\
$+2 S D$ & 2.94 & 3.67 & 4.24 \\
\hline
\end{tabular}

Note. All other covariates held at the sample mean. Low $=-1 / 2 S D$, High $=+1 S D$, Very High $=+2 \mathrm{SD}$. 